EPJ Web of Conferences 106, 04004 (2016)

DOI: $10.1051 /$ epjconf/201610604004

(C) Owned by the authors, published by EDP Sciences, 2016

\title{
A Comprehensive Approach to Determination of Nuclear Data of Unstable Nuclei
}

\author{
Satoshi Chiba ${ }^{1,2, a}$, Katsuhisa Nishio ${ }^{2}$, Yohihiro Aritomo ${ }^{1}$, Hiroyuki Koura ${ }^{2}$, Osamu Iwamoto ${ }^{2}$, Hiroyuki \\ Makii $^{2}$, Ichiro Nishinaka ${ }^{2}$, and Kentaro Hirose ${ }^{2}$ \\ ${ }^{1}$ Research Laboratory for Nuclear Reactors, Tokyo Institute of Technology, 2-12-1 Ookayama, \\ Meguro-ku, Tokyo 152-8550, Japan \\ ${ }^{2}$ Advanced Science Research Center, Japan Atomic Energy Agency, 2-4 Shirakata Shirane, Naka-gun, \\ Tokai, Ibaraki 319-1195, Japan
}

\begin{abstract}
A comprehensive approach to determine nuclear data of unstable nuclei will be described. It consists of a measurement of fission and capture cross sections, mass distribution of fission fragments (independent fission yields) and number of prompt fission neutrons by the method of surrogate reactions. A multi-dimensional Langevin model is being developed to estimate the independent fission yields theoretically. Furthermore, the $\beta$ decay properties of the fission fragments, almost all are neutron-rich unstable nuclei, are investigated systematically by improving the gross theory of $\beta$ decay, which will yield information on the decay heat and delayed-neutron data
\end{abstract}

\section{Introduction}

Nuclear data for unstable nuclei are necessitated more and more for advanced reactor dosimetry as the burnup of nuclear reactors increases. Fission and capture cross sections of minor actinides, and capture cross sections of fission fragments will play important roles not only in dosimetry but in disposal of nuclear waste, and they also affect performances of high-burnup reactors and subcriticality of accelerator-driven systems (ADS) for nuclear transmutation. Delayed neutron data are also key information to evaluate kinetics and reactivity of nuclear reactors and other nuclear facilities including ADS. Estimation of the delayed-neutron data requires a comprehensive knowledge on the fission of relevant nuclei, namely how the fission fragments are populated in the nuclear chart and how they decay toward stable nuclei. However, there are still many problems in nuclear data of unstable nuclei because experiments are difficult, and the physics of fission and neutron-rich nuclei are complicated. These are important issues on the front line of nuclear physics.

In our approach, we will conduct the following comprehensive and systematic research to improve our experimental methodology and knowledge on nuclear data of unstable nuclei as a collaboration

\footnotetext{
${ }^{a}$ Corresponding author: chiba.satoshi@nr.titech.ac.jp
}

This is an Open Access article distributed under the terms of the Creative Commons Attribution License 2.0, which permits unrestricted use, distribution, and reproduction in any medium, provided the original work is properly cited. 
between Tokyo Tech. and JAEA (Japan Atomic Energy Agency) supported by MEXT (Ministry of Education, Culture, Sports, Science \& Technology in Japan):

1. Development of experimental methodology for measurement of neutron cross sections of unstable nuclei and mass distributions of fission fragments (MDFF) by a method of surrogate reactions. It will yield cross section data for minor actinides and fission products, which are hard to obtain by other methods in general.

2. Development of a multi-dimensional Langevin model to estimate the independent fission yield (not only mass yield but also isotopic distributions), which is tuned to reproduce the MDFF data obtained in item 1.

3. Gross theory of $\beta$-decay will be extended to reproduce available up-to-date data for the half lives of $\beta$-decay, spectra of $\beta$-rays and $\gamma$-rays, and delayed neutron emission probabilities of relevant nuclei. Then, it will be used to estimate the decay heat, delayed-neutron emission and emission of antineutrinos (including their spectra) of nuclei for which no data are available.

4. A statistical decay model based on the Hauser-Feshbach formula will be fully employed in conjunction with items 2 and 3 [1]. In item 2, it will be used to estimate prompt neutron emission that modifies the primary fission yields (before the emission of prompt neutrons) to independent ones. In item 3, the statistical decay model estimates the delayed-neutron emission probability and spectra of delayed neutrons.

The following includes the present status of the project, including the outline, development of equipment, theory development and some of the first results will be described mainly for items 1 and 2 only due to shortage of the space.

\section{Outline of the Project}

\subsection{Development and Verification of the Method of Surrogate Reactions}

Measurement of reaction properties of unstable nuclei requires preparation of sufficient amounts of radioactive materials for the target and experiments at high-intensity neutron source facilities such as n-TOF [2] and J-PARC [3]. However, such experiments are extremely difficult or practically impossible, in general. An alternative is to employ the method of surrogate reactions, in which nucleon transfer reactions on stable nuclei are used to populate the same compound nuclei as the desired neutroninduced reactions on unstable nuclei, and decay properties such as branching ratios of the populated nuclei are determined $[4,5]$. In the surrogate ratio method, such measurements are performed for a pair of compound nuclei, and one of them is used as the reference to determine the neutron-induced cross sections of the other, which are supposed to be unknown. By taking the ratio of branching ratios, it can be shown that many unknown uncertainties such as the difference of the spin-parity $\left(\mathrm{J}^{\pi}\right)$ distributions of the populated compound nuclei and effects of the pre-equilibrium and breakup reactions between the neuron-induced reactions and surrogate reactions cancel out [6]. All we have to control is that the two target nuclei used in the surrogate ratio method have to have the same reaction mechanisms and the structure of relevant nuclei must be equivalent as well so that the $\mathrm{J}^{\pi}$ distributions must be considered to be equivalent. Such equivalence can be verified by a dynamical model [7] and a coupledchannels theory calculation [8], and is considered to be valid unless the equivalent neutron energy is not too small, where the competition for neutron emission is strongly dependent on the $\mathrm{J}^{\pi}$ of the populated nuclei. Similarly, the surrogate method will not work correctly if the excitation energy of the populated nuclei is too large because the spin distribution will become drastically different from that of desired neutron-induced reactions [8]. Observables depending on the spin of populated nuclei are proposed in Ref. [9] 

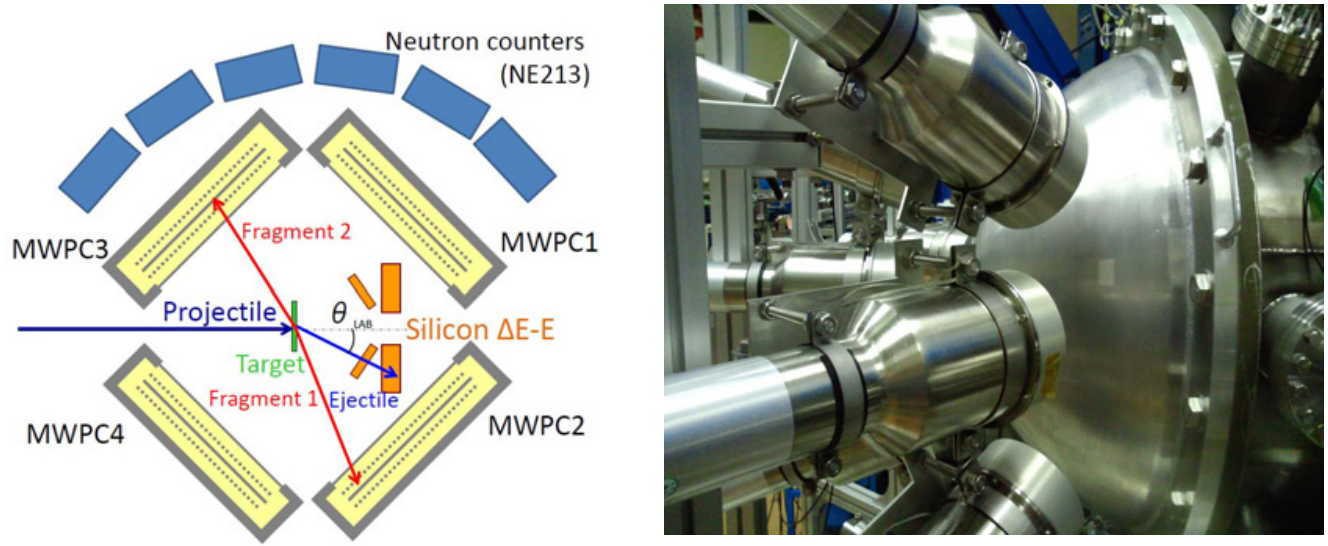

Figure 1. Left: schematic layout of various detectors used in the method of surrogate reactions. Right: a Photograph of a vacuum chamber surrounded by NE213 neutron counters.

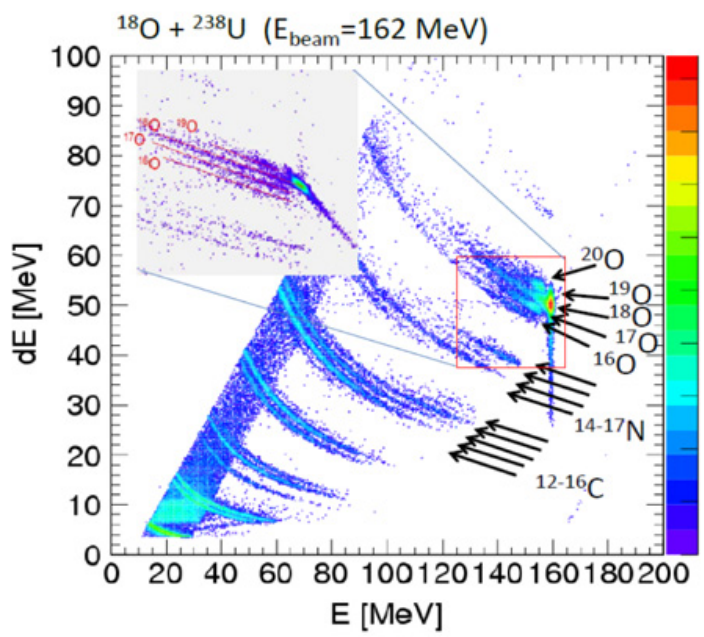

Figure 2. A typical particle-identification spectrum obtained by the $\Delta \mathrm{E}-\mathrm{E}$ counter for a reaction ${ }^{18} \mathrm{O}(162 \mathrm{MeV})$ on a ${ }^{238} \mathrm{U}$ target.

\subsubsection{Measurements of Fission Cross Sections, Mass Distribution of Fission Fragments and Number of Prompt Neutrons per Fission}

The left panel of Fig. 1 shows a schematic layout of detectors used in the method of surrogate reactions to determine fission cross sections: a Si $\Delta \mathrm{E}$-E detector for identification of outgoing particles, $4 \mathrm{MWPC}$ 's (Multi-wire proportional counters) for detection of fission fragments and neutron detectors to measure number of prompt fission neutrons. A photograph of the vacuum chamber containing the target, the $\mathrm{Si}$ $\triangle \mathrm{E}-\mathrm{E}$ detector and 4 MWPC's surrounded by neutron detectors (NE213) is shown in the right panel of Fig. 1. The front surface of the vacuum chamber was made with a thin Al to prevent scattering of prompt fission neutrons. Inside the vacuum chamber, the projectile hits the target and turns into various kinds of ejectiles as a result of nucleon-transfer reactions, and they are identified by the $\Delta \mathrm{E}-\mathrm{E}$ detector, which consists of a $75 \mu \mathrm{m}$ and a $300 \mu \mathrm{m}$-thick Si counters. The typical $\Delta \mathrm{E}$-E spectrum is displayed in Fig. 2 to indicate how the ejectiles and their energies could be identified. Number of 

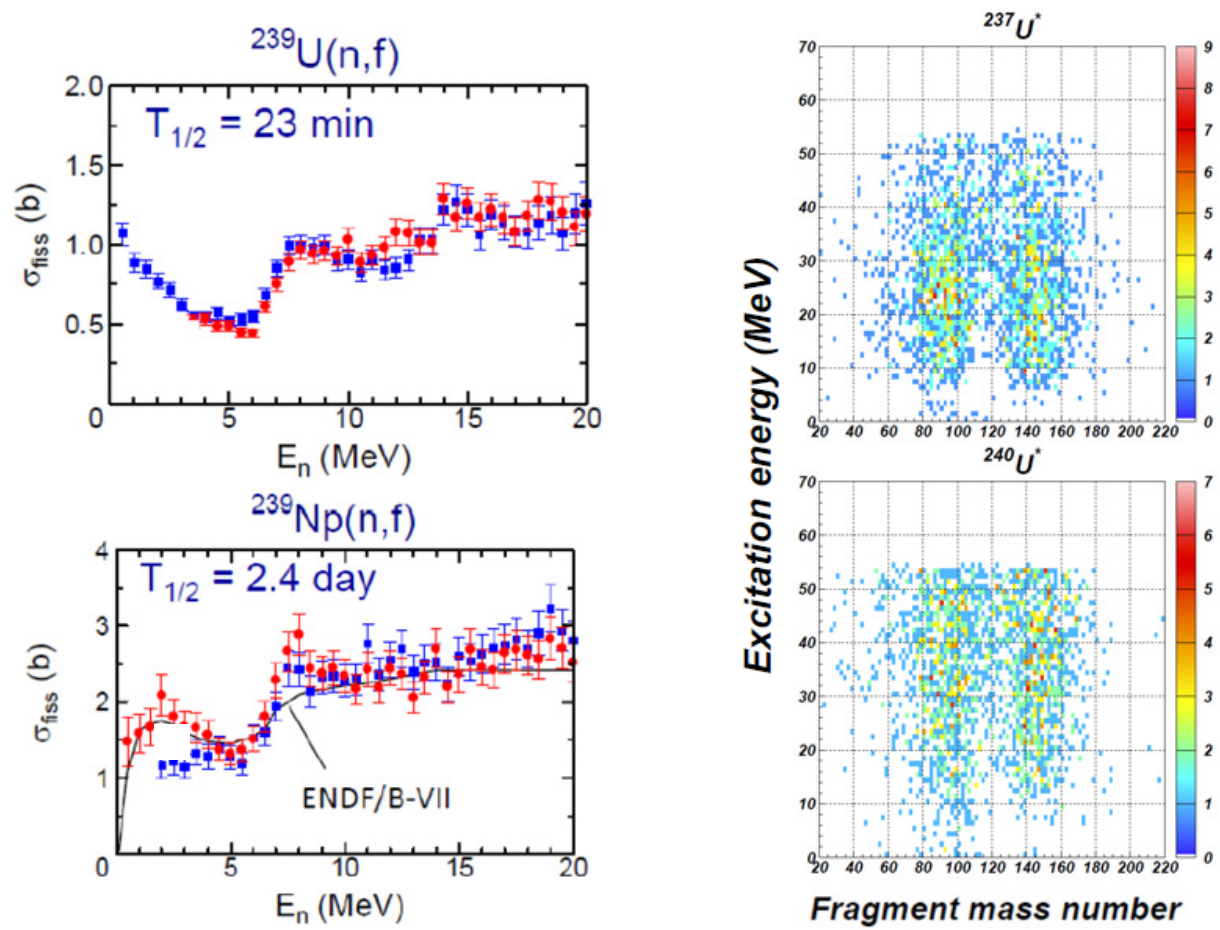

Fragment mass number

Figure 3. Left: neutron-induced fission cross sections of ${ }^{239} \mathrm{U}\left(\mathrm{T}_{1 / 2}=23.5 \mathrm{~min}\right.$.) and ${ }^{239} \mathrm{~Np}\left(\mathrm{~T}_{1 / 2}=2.4 \mathrm{~d}\right)$ determined by the method of surrogate reactions (preliminary). The two symbols denote results obtained by using different reactions as the "reference" (right). Fission fragment distribution as two-dimensional data on excitationenergy (vertical axes, in $\mathrm{MeV}$ ) and mass number of fission fragments (horizontal axes) from ${ }^{237} \mathrm{U}$ (top panel) and ${ }^{240} \mathrm{U}$ (bottom panel) isotopes are populated by the surrogate reactions using ${ }^{18} \mathrm{O}$ beam on ${ }^{238} \mathrm{U}$. All the results in these figures are still preliminary.

particles are indicated by a collar contour in the $\mathrm{z}$ scale (maximum is normalized to 1 ). This spectrum was obtained for a combination of a projectile ${ }^{18} \mathrm{O}\left(\mathrm{E}_{\mathrm{lab}}=162 \mathrm{MeV}\right)$ on a ${ }^{238} \mathrm{U}$ target. We can notice that many isotopes of oxygen are identified as well as their outgoing energies. Therefore, they will signal that many isotopes of $\mathrm{U}$, such as $\mathrm{A}=240,239,238,237$ and 236 were populated at certain excitation energies. Furthermore, we notice that isotopes of $\mathrm{N}$ and $\mathrm{C}$ are also detected as ejectiles, denoting that various isotopes of $\mathrm{Np}$ and $\mathrm{Pu}$ are also populated in the same experiment. This is the advantage of employing the surrogate experiment with a heavy-ion projectile. By selecting a specific reaction channel, e.g. ${ }^{238} \mathrm{U}\left({ }^{18} \mathrm{O},{ }^{16} \mathrm{O}\right)$ reaction, we can populate a compound nucleus of ${ }^{240} \mathrm{U}$ at various excitation energies. The decay branching ratio from it is then determined by the ratio of count rates of the MWPC and $\triangle \mathrm{E}-\mathrm{E}$ counters. This ratio is related to the branching ratio of the fission channel from the ${ }^{239} \mathrm{U}$ (n, fission) reaction. Similar ratios can be also obtained by ${ }^{235} \mathrm{U}\left({ }^{18} \mathrm{O},{ }^{16} \mathrm{O}\right){ }^{237} \mathrm{U}$ and ${ }^{235} \mathrm{U}\left({ }^{18} \mathrm{O},{ }^{19} \mathrm{O}\right){ }^{236} \mathrm{U}$ reactions, which are related to ${ }^{236} \mathrm{U}$ (n,fission) and ${ }^{235} \mathrm{U}$ (n,fission), respectively, for which neutron-induced cross sections are available. Then, by adopting the known fission cross sections of ${ }^{236} \mathrm{U}$ and ${ }^{235} \mathrm{U}$, we can determine the neutron-induced fission cross section of ${ }^{239} \mathrm{U}$ by taking the ratio of these branching ratios. Cross sections for ${ }^{239} \mathrm{U}$ (n,fission) and ${ }^{239} \mathrm{~Np}$ (n,fission) thus determined are displayed in the left panel of Fig. 3. They are still preliminary, and the error bars indicate the statistical uncertainty alone. We estimate that the overall systematic uncertainty is $20 \%$, at most. Still, the method of surrogate reaction will be able to populate a number of useful data because the accuracy of the model prediction of fission cross sections would be close to a factor of 2 if no experimental data exists. 


\section{$15^{\text {th }}$ ISRD}

In the right panel of Fig. 3, mass distributions of fission fragments (MDFF) emitted from ${ }^{237} \mathrm{U}$ and ${ }^{240} \mathrm{U}$ from various excitation energies, determined from the surrogate reaction ${ }^{18} \mathrm{O}+{ }^{238} \mathrm{U}$, are shown as a contour map. Actual examples of MDFF will be shown later for the case of ${ }^{240} \mathrm{U}$. Similarly, the number of prompt fission neutrons from a number of excited major- and minor-actinides can be determined from the count rate of the NE213 neutron counters surrounding the target chamber. These results are only a part of the data we can obtain from the method of surrogate reactions, but already indicate that reaction properties of short-lived unstable nuclei can be deduced from it. All of these results will be finalized in near future.

\subsubsection{Determination of Neutron Capture Cross Sections by the Surrogate Method: A Validation}

Determination of the neutron capture cross sections of unstable nuclei is one of the most important subjects of current nuclear-data activities because accurate dosimetry and burnup calculations, and research in nucleosynthesis in stars and r-process require such information as key input data. Accuracy of the theoretical estimation could be off by a factor of 10 in many cases if no experimental data exists. However, determination of the neutron-induced capture cross sections is much more difficult compared to the success in obtaining neutron-induced fission cross sections by the method of surrogate reactions. It is because of the fact that the competition to neutron emission has a strong dependence on the spinparity of the compound nucleus at energy region where the capture reaction is important for application (corresponding to incident neutron energy below $1 \mathrm{MeV}$ ). Above this energy, on the contrary, the capture cross section becomes drastically small, thus determination of it would become difficult also. The results for fission reactions shown above were obtained for energy region where the competition to neutron emission is not very sensitive to the spin parity, while the fission cross section is not too small as well. Therefore, we have chosen the pair of target nuclei to be used in the surrogate ratio method carefully to have the spin-parity configuration as close as possible to each other to validate the surrogate method applied to determine neutron capture cross sections. Targets of ${ }^{155} \mathrm{Gd}$ and ${ }^{157} \mathrm{Gd}$ were selected because both have the ground-state spin parity of $3 / 2^{-}$, and if two neutrons are transferred to them via the $\left({ }^{18} \mathrm{O},{ }^{16} \mathrm{O}\right)$ reaction, the populated compound nuclei are ${ }^{157} \mathrm{Gd}$ and ${ }^{159} \mathrm{Gd}$, and they again have the ground-state spin parity of $3 / 2^{-}$. Of course, the neutron will be emitted from the compound nuclei to populate ${ }^{156} \mathrm{Gd}$ and ${ }^{158} \mathrm{Gd}$, both having $0^{+}$ground-state spin parity.

A schematic setup of detectors to determine neutron capture cross sections by the surrogate method is shown in Fig. 4. In this figure, the ${ }^{18} \mathrm{O}$ beam will impinge from the bottom and interact with the target located at the center of a vacuum chamber where nucleon transfer reactions will take place. Emitted $\gamma$-rays are detected by two $\mathrm{LaBr}_{3}(\mathrm{Ce})$ scintillators surrounded by an annular BGO scintillator for Compton suppression. These detectors are located face-to-face surrounding the target.

Performance of each $\mathrm{LaBr}_{3}(\mathrm{Ce})$ detector was determined by measuring $\gamma$-rays from standard sources $\left({ }^{60} \mathrm{Co}\right.$ and $\left.{ }^{88} \mathrm{Y}\right)$ and those from ${ }^{27} \mathrm{Al}(\mathrm{p}, \gamma)$ reactions at $\mathrm{E}_{\mathrm{p}}=992$ and $2046 \mathrm{keV}$, where $\gamma$-rays with wellknown intensities are emitted. The energy resolution of the $\mathrm{LaBr}_{3}(\mathrm{Ce})$ detector was observed to be $2.1 \%$ at $1.3 \mathrm{MeV}$. The surrogate experiment was carried out with ${ }^{155} \mathrm{Gd}$ and ${ }^{157} \mathrm{Gd}$ targets $(92 \%$ enriched in each), $0.9 \mathrm{mg} / \mathrm{cm}^{2}$ in thickness and $10 \mathrm{~mm}$ in diameter. The bombarding energy of ${ }^{18} \mathrm{O}$ was $153 \mathrm{MeV}$, and outgoing particles were detected by the Si $\Delta \mathrm{E}-\mathrm{E}$ detector for particle and energy identification similar to the fission experiment.

Left panels of Fig. 5 show deexcitation $\gamma$-ray spectra from ${ }^{157} \mathrm{Gd}$ and ${ }^{159} \mathrm{Gd}$ obtained by setting gates on ${ }^{16} \mathrm{O}$ particles with kinetic energies corresponding to the excitation energies for ${ }^{157} \mathrm{Gd}$ and ${ }^{159} \mathrm{Gd}$ between 3 and $14 \mathrm{MeV}$. Continuum $\gamma$-rays up to about $8 \mathrm{MeV}$ arise from the deexcitation of highly excited states in ${ }^{157} \mathrm{Gd}$ and ${ }^{159} \mathrm{Gd}$ populated by the ${ }^{155,157} \mathrm{Gd}\left({ }^{18} \mathrm{O},{ }^{16} \mathrm{O}\right)$ two-neutron transfer reactions. The decay branching ratio of the $(\mathrm{n}, \gamma)$ process $P_{\gamma}\left(E_{X}\right)$ at excitation energy $E_{x}$ can be determined from the ratio of the number of $\gamma$ rays in coincidence with ${ }^{16} \mathrm{O}$ particles $\left(N_{O-\gamma}\left(E_{X}\right)\right)$ to the number of ${ }^{16} \mathrm{O}$ 


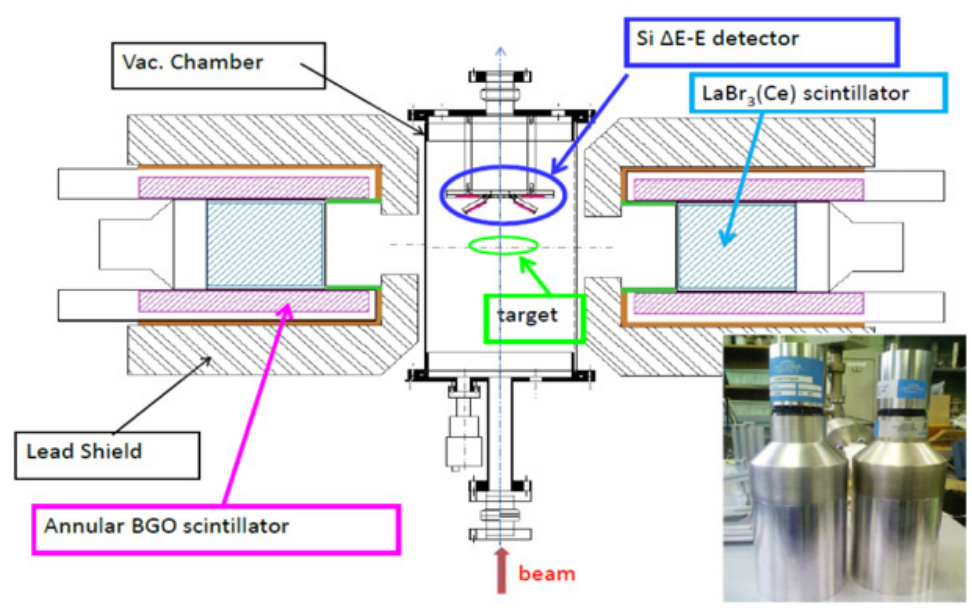

Figure 4. A setup to determine neutron capture cross sections by the surrogate method. A photograph of the $\mathrm{LaBr}_{3}(\mathrm{Ce})$ detector $(101.6 \mathrm{~mm}$ in diameter and $127 \mathrm{~mm}$ in length) is shown in the right-bottom part.
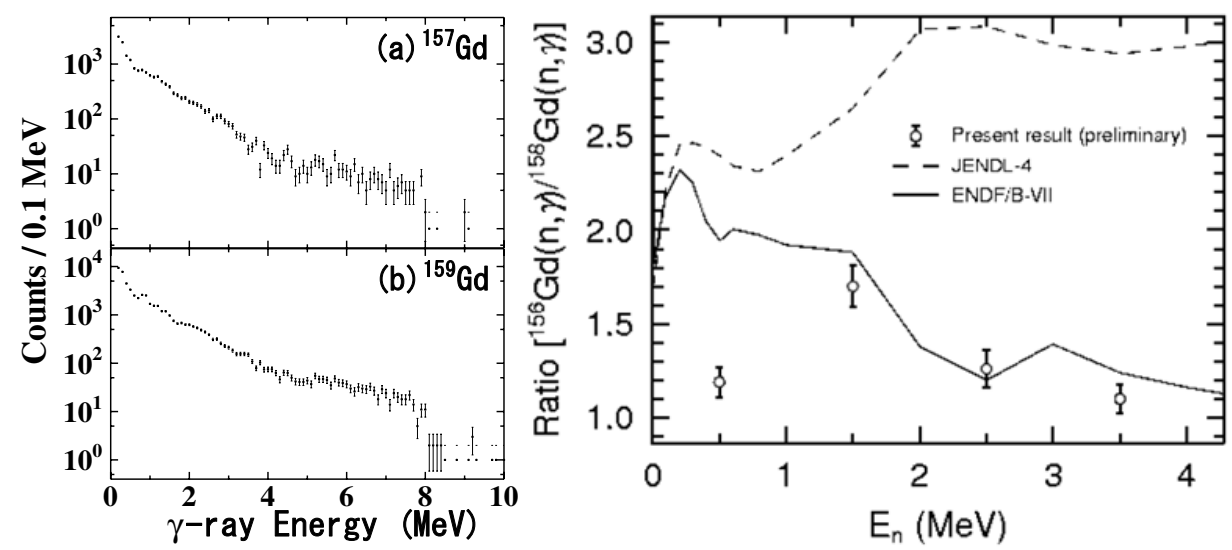

Figure 5. Left: deexcitation $\gamma$-ray energy spectra emitted from ${ }^{157} \mathrm{Gd}$ and ${ }^{159} \mathrm{Gd}$ at excitation energy region between 3 and $14 \mathrm{MeV}$ populated by $\left({ }^{18} \mathrm{O},{ }^{16} \mathrm{O}\right)$ reactions on ${ }^{155} \mathrm{Gd}$ and ${ }^{157} \mathrm{Gd}$ targets (right). Ratio of the neutron capture cross sections for ${ }^{156} \mathrm{Gd}(\mathrm{n}, \gamma)$ and ${ }^{158} \mathrm{Gd}(\mathrm{n}, \gamma)$ reactions determined from the present method (open circles) compared with data in ENDF/B-VII (solid line) and JENDL-4 (dashed line).

particles $\left(N_{O}\left(E_{x}\right)\right)$

$$
P_{\gamma}\left(E_{X}\right)=\frac{N_{O-\gamma}\left(E_{X}\right)}{N_{O}\left(E_{X}\right)}
$$

$N_{O-\gamma}\left(E_{X}\right)$ can be obtained by using the weighting function technique [10], which requires complete knowledge of the response functions for the $\mathrm{LaBr}_{3}(\mathrm{Ce})$ spectrometers.

The right panel of Fig. 5 denotes the ratio of the neutron capture cross sections for ${ }^{156} \mathrm{Gd}(\mathrm{n}, \gamma)$ to that of ${ }^{158} \mathrm{Gd}(\mathrm{n}, \gamma)$ reactions after converting the excitation energy of the compound nuclei to corresponding incident neutron energy. The presently determined results are noted by open circles, where errors designate statistical results alone. The solid line shows an equivalent ratio calculated from data stored in ENDF/B-VII, while the dashed line was calculated from JENDL-4. As we can imagine, the present data at the lowest energy point $(0.5 \mathrm{MeV})$ is lower than the evaluated data due to strong competition by 
the neutron emission, which is strongly dependent on the spin and parity. Therefore, we cannot rely on this data point. However, our results are in marked agreement with ENDF/B-VII above $1.5 \mathrm{MeV}$, which in turn are in good agreement with data measured directly using neutrons. Hence, data obtained by the surrogate method will be useful to validate or estimate neutron-induced capture cross sections around 1 to $3 \mathrm{MeV}$. Then, the parameters in the statistical model (e.g., giant-dipole resonance parameters and level density parameters) can be determined at this energy region to reproduce the data from surrogate method, then the model prediction can be extended to lower and higher energy regions to estimate the neutron capture cross sections in the entire energy region. Therefore, it was verified that the surrogate method can be utilized to determine the neutron-induced capture cross sections of unstable nuclei if enough caution is paid on the condition with which this method will potentially yield the correct results.

\subsection{Development of a Multi-dimensional Langevin Model to Estimate the Independent Fission Yield}

A dynamical model of nuclear fission is being developed based on the fluctuation-dissipation theorem formulated as Langevin equations [11]. The Langevin equations to describe time evolution of collective variables $q_{i}$ expressing nuclear shape during the fission $(i=1,2,3$ : center distance between 2 fragments $z$, their deformations and mass asymmetry $\alpha$ ) and their conjugate momenta $p_{i}$ are given as the following set of equations:

$\frac{d q_{i}}{d t}=\left(m^{-1}\right)_{i j} p_{j}, \quad \frac{d p_{i}}{d t}=-\frac{\partial V}{\partial q_{i}}-\frac{1}{2} \frac{\partial}{\partial q_{i}}\left(m^{-1}\right)_{j k} p_{j} p_{k}-\gamma_{i j}\left(m^{-1}\right)_{j k} p_{k}+g_{i j} R_{j}(t), \quad(i, j=1,2,3)$

where summation over repeated indices is assumed. The symbol $V$ denotes the potential energy surface as a function of the 3 nuclear shape parameters, and is calculated by the 2-center shell model [12], $R_{j}(t)$ being the random force having the nature of white noise (Markov process), $g_{i j}$ strength of the random force. The transport coefficients $m_{i j}$ (inertia tensor) and $\gamma_{i j}$ (friction tensor) were calculated by a Werner-Wheeler's hydrodynamical model and wall-and-window formula, respectively [13, 14]. The $g_{i j}$ and $\gamma_{i j}$ are related to each other by the Einstein relation $g_{i k} E g_{k j}=T \gamma_{i j}$, where $T$ denotes the temperature of the fissioning nuclei. This condition fixes $g_{i j}$. Nucleon transfers between the fragments is determined by a diffusion-type equation with a drift term for neutron and proton separately. In this way we can determine the isotopic distribution (the primary fission yield before emission of prompt neutrons) of fission fragments as well as the mass distribution to be compared with the results obtained by the surrogate method. Quantum effects are included in the potential energy surface, namely, the shell effects by the Strutinski method, and the pairing effects by the BCS model [15]. An example of a potential energy surface calculated in this method is displayed in Fig. 6 as a function of center-of-mass (C.M.) distance between the two fragments $Z$ and mass asymmetry parameter $\alpha$, which is defined as $\left(A_{1}-A_{2}\right) / A$. Here, $A_{1}$ denotes a mass number of fragment $1, A_{2}$ that of fragment 2 , and $A=A_{1}+A_{2}$.

Starting from a configuration around the ground state, the Langevin trajectories are generated for many events. An example of such trajectories is shown in Fig. 6 as a white line superimposed on the potential energy surface. The fission events are determined in our model by tracing the time evolution of the trajectories in the deformation space, and is defined as the case that a trajectory overcomes the scission point (beyond which 2 fragments do not exchange particles any more) on the potential energy surface at which we can determine the mass, isotopic and kinetic energy distributions of the primary fission fragments (before emission of prompt neutrons from the fragments). The pre-scission neutron emission is taken into account in the Langevin calculation based on the statistical model.

Dependence of the calculated results on the model parameters such as the friction coefficients and neck parameter were investigated. It was found that overall strength of the friction coefficients had little influence on the final MDFF. On the other hand, the neck parameter $\varepsilon$ modifies the peak-to-valley ratio of the MDFF and total kinetic energy (TKE) of the fission fragments. We found that $\varepsilon=0.35$ is the 


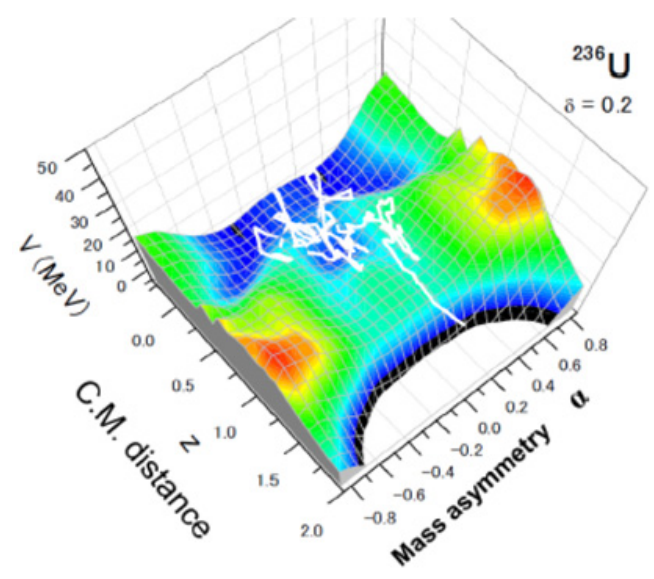

Figure 6. Example of a landscape of a multi-dimensional potential energy $V$ calculated by the 2-center shell model and a representative Langevin trajectory (white line).
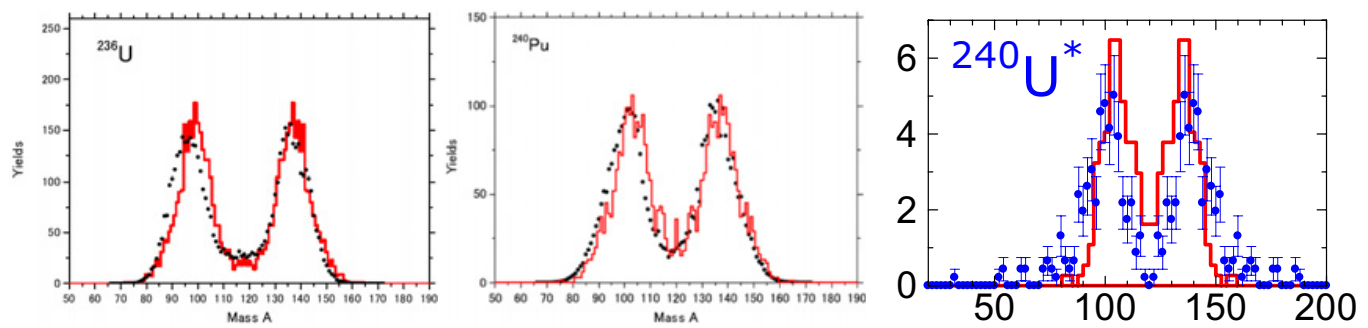

Figure 7. Mass distribution of fission fragments (MDFF) of ${ }^{236} \mathrm{U}$ (leftmost panel), ${ }^{240} \mathrm{Pu}$ (center panel) and ${ }^{240} \mathrm{U}$ (rightmost panel) at excitation energy of $20 \mathrm{MeV}$. The horizontal axes denote the mass number of fission fragments, while the vertical axes designate relative fission yields. Data for ${ }^{240} \mathrm{U}$ obtained (squares) by the surrogate method of the present work are still preliminary. In each figure, the data are normalized to give same areas.

best value to describe the overall feature of the fragment mass and TKE distribution. Typical results of our calculations for MDFF are given in Fig. 7. The squares in the leftmost $\left({ }^{236} \mathrm{U}\right)$ and center $\left({ }^{240} \mathrm{Pu}\right)$ panels denote data given in JENDL based on experimental information, while the histogram denotes results of our Langevin calculations. These two figures are shown to validate our approach to calculate the MDFF by the Langevin approach, while the rightmost figure shows a similar comparison of the data obtained from the surrogate method by the ${ }^{238} \mathrm{U}\left({ }^{18} \mathrm{O},{ }^{16} \mathrm{O}\right){ }^{240} \mathrm{U}^{*}$ reaction (squares) and our theoretical predictions (histogram). The nucleus ${ }^{240} \mathrm{U}$ is a compound one in the $\mathrm{n}+{ }^{239} \mathrm{U}$ reaction, for which a target of ${ }^{239} \mathrm{U}\left(\mathrm{T}_{1 / 2}=23 \mathrm{~min}\right.$.) must be prepared. Of course, such a measurement is impossible. Only the surrogate method can produce this kind of nuclear data. The peak positions are reproduced quite well, while the width of the distribution is slightly underestimated. However, this may come from the improper treatment of the experimental data, which are still not final. Nevertheless, the present approach, including both a novel experimental technique (the surrogate method) and theoretical frameworks, will be proven to be a potentially powerful tool to determine nuclear data of unstable nuclei.

\section{Conclusions and Outlook}

Determination of neutron cross sections of unstable nuclei and nuclei for which preparation of targets is extremely difficult for direct measurements using neutron beams is one of the most challenging issues 


\section{$15^{\text {th }}$ ISRD}

in reactor dosimetry and nuclear data in general. We propose a novel method based on the surrogate technique, in which the same compound nuclei as the desired neutron-induced reactions are populated by other reactions such as neutron-transfer reactions, and the decay properties of them are measured. From this information, we can determine the decay branching ratio of the compound nuclei, and by taking a proper ratio to the same quantity in those reactions where the neutron cross sections are known, we can determine neutron cross sections of these target nuclei. A special feature of our method is to use a heavy ion beam $\left({ }^{18} \mathrm{O}\right)$ as the projectile, and populate many compound nuclei at the same time. So far, we have come to a point that we can determine neutron fission cross sections with a systematic error within $20 \%$, while determination of the neutron capture cross section needs much caution. Theories such as Langevin equation and gross theory (which are not described here) are also important pieces of our comprehensive approach.

The present study includes the results of "Development of a Novel Technique for Measurement of Nuclear Data Influencing the Design of Advanced Fast Reactors" entrusted to Japan Atomic Energy Agency (JAEA) by the Ministry of Education, Culture, Sports, Science and Technology of Japan (MEXT), and also "Comprehensive study of delayed-neutron yields for accurate evaluation of kinetics of high-burn up reactors" entrusted to Tokyo Institute of Technology by MEXT.

\section{References}

[1] O. Iwamoto, J. of Nucl. Sci. Tech. 44, 687 (2007)

[2] "n-TOF", https://ntof-exp.web.cern.ch/ntof-exp/

[3] M. Igashira, Y. Kiyanagi and M. Oshima, Nucl. Instrum. Meth. in Phys. Res. A 600, 332 (2009)

[4] J.E. Escher, T.T. Burke, F.S. Dietrich, N.D. Scielzo, I.J. Thompson, W. Younes, Rev. of Mod. Phys. 84, 353 (2012)

[5] S. Chiba, K. Nishio, H. Makii, et al., Nuclear Data Sheets (in press)

[6] S. Chiba and O. Iwamoto, Phys. Rev. C 81, 044604-1 (2010)

[7] Y. Aritomo and S. Chiba, Phys. Rev. C 84, 024602-1-10 (2011)

[8] K. Ogata, S. Hashimoto and S. Chiba, J. Nucl. Sci. Tech. 48, 1337 (2011)

[9] S. Chiba, O. Iwamoto and Y. Aritomo, Phys. Rev. C 84, 054602-1 (2011)

[10] R.L. Macklin, J.H. Gibbons, Phys. Rev. 159, 1007 (1967)

[11] Y. Aritomo and S. Chiba, Phys. Rev. C 88, 044614-1(2013)

[12] J. Maruhn and W. Greiner, Z. Physik 251, 1972, 431 (1972)

[13] K.T.R. Davies, A.J. Sierk, and J.R. Nix, Phys. Rev. C 13, 2385 (1976)

[14] A.J. Sierk and J.R. Nix, Phys. Rev. C 21, 982 (1980)

[15] S.G. Nilsson and I. Ragnarsson, "Shapes and shells in nuclear structure", Cambridge university press, 1995 